\title{
The Formation, Growth, and Habit of Crystals
}

\author{
Modern Views of Crystallography
}

\author{
By Paul Gaubert, D. Sc, Assistant in Mineralogy at the Natural History Museum, iParis
}

A CRYstal arouses the interest of the observer not only by the regularity of its forms, the perfection of its surfaces and angles, its transparency, and its bril liancy, but also by the manner in which it grows heals its wounds, is dissolved, and modifled under th influence of the inclosing medium. To some author the crystal, from certain points of view, appear analogous to living forms, and seems to undergo a sort of evolution.

Its formation, its growth, the variations of the face under the influence of the inclosing medium, have been the object of numerous researches which have greatly modified our conceptions regarding them. The purpose of this article is to show the present state of our knowledge concerning these diverse and interest ing questions of crystallogeny.

As early as the seventeenth century Leeuwenhoek, who examined under the microscope everything that in his time lent itself to this line of observation, followed the formation and growth of the crystals of various substances (as sugar, tartar, sea salt, etc.). He was led to conclude that the cubic crystals of se salt are formed of other minute cubes, themselve made up from cubes, the existence of which one ha to accept through analogy with what is seen, since they are invisible under any magnifying power. Later, Baker, Ledermüller, and others also examined under the microscope the branched and varied form that appear when a substance crystallizes on a sheet of glass; but it is to Nicholas Le Blanc that we ow the flrst systematic and effective researches in crystal genesis, and particularly in the variation of the form. In his very interesting work; "De la Cristallotechnie," he gives methods for the preparation of crystals, an in particular does he set forth the process of renewing the solution, of "feeding" the growing solids that they may attain a relatively considerable size.

In what form do we see the crystal with the aid of the highest magnifying power? Does it present from the flrst the form that it will have later? The biologists were the flrst to take up the matter of the formation of the crystalline "germ"; that is, of the formation of the crystalline "germ"; that is, of th becomes visible; and most of them have admitted th existence of an embryonic state, or a state in whic the constitution and form are different from that of

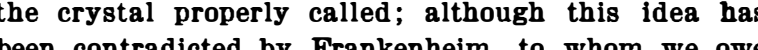
by Frankenheim, to whom we ow numerous crystallogenic observations. Vogelsang, in 1867, took it up again and made numerous ingenious and varied experiments to show its correctness. His
observations are generally exact, but he has unfortuobservations are generally exact, but he has unfortunately misinterpreted them. To show the embryonic state of the crystal, Vogelsang tried to make the bodies crystallize under special conditions with the purpose of retarding their formation so as to enable him to observe all the steps of development. With this purpose in view he added to sulphur solution viscous body, Canada balsam. There were produced little spheres, to which Vogelsang gave the name of globulites, and which were thought to represent an embryonic stage. These globulites unite to form par ticular groups, each of which has received a specia name, and at the expense of which the crystal would be produced only at a later stage.

Moreover, Vogelsang rests his experimental re searches on observations made with crystallites of varied forms existing in a few rocks rich in silica an more or less vitreous, and in the slags of blast furnaces; but as was later shown by $M$. $O$. Lehmann, who made numerous researches on the formation of crystals, these globulites are but drops supersaturated with sulphur, and consequently have nothing in com mon with the crystalline state.

Brame, as well as Vogelsang, studied sulphur, but in a molten condition. He observed little supermelted drops (utricules) to which he attributes a considerable role in crystallization. His ldeas differ from those of Vogelsang, but nothing in his experiment substantiates the existence of an embryonic state.

The observations of M. O. Lehmann have shown that the crystal possesses from the beginning a forn identical with that which it has when it has attaine larger dimensions. T. V. Richard and E. H. Archibald have employed the cinematograph to follow out the formation of the crystal, and obtained only figure of completely formed individuals.

I myself have made a great many experiments, and have always found that the first visible particle had

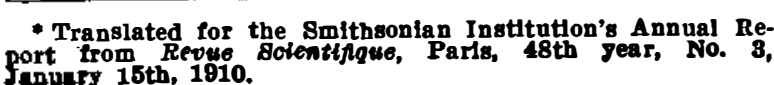

all the properties of the crystal. It is, nevertheless, not to be disputed that in some cases there takes place what Vogelsang and his predecessors have observed with sulphur or other bodies, but who worked with supersaturated drops or amorphous particles, or little spherulites of unstable form, which later underwent modifications into more stable forms, and the normal development of which can then be followed.

Nevertheless, in spite of the observations of Frankenheim, 0 . Lehmann, and others, the idea of the embryonic state of the crystal has not disappeared from science, and the hypothesis of Vogelsang, supported by De Schoen, Cartaud, and others, resting on misinterpreted observations, still finds some credit.

When the crystal is once formed-that is, becomes visible under the microscope-how does it grow? Several cases may be presented: First, the mother liquid is in a state of rest, the cooling or evaporation is extremely slow, and the crystalline particles are built p by diffusion alone. In this case the growth is too low to be constantly followed under the microscope. In the second case the liquid is cooled or evaporated with such rapidity that the quantity of matter deposited on the crystal produces an enlargement microscopically visible. Movements in the liquid are thereby set up. It is an established fact that currents called "currents of concentration" passing over a crystal, deposit a thin coating of substance, followed by a second, and so on, until, for example, one can see on a crystal of lead nitrate, having a diameter of half a milimeter, as many as twelve of these succesinterrupted and the crystal examined any observed face would not be a plane, but would show a sort of step arrangement, of which the highest step would in. dicate the point of contact of the current of dedicate the

These successive déposits have no interspaces and the crystal may be perfectly transparent: If the fluence of two or of several currents of concentration; the corresponding coatings laid upon it start from different points in the periphery and may not be of the same thickness. Ordinarily they do not join exactly at their point of meeting. In this way arê then produced inclusions and the crystal is no longer transparent, but becomes milky. On a glass plate it is easy to produce at will a transparent or milky crystal of lead nitrate. In the experiment it is necessary to agitate the crystal very slightly with a needle in order to subject it to the influence of one or several currents.

These concentration currents produce other peculiarities (vicinal faces, etc.), which it would take too long to describe in detail. I shall conflne myself to calling attention to the influence they may have upon the faces of the crystal. The introduction of matter to one face only of a crystal causes it to develop unequally, and since all crystals of the same bath or magma are not influenced in the same way, they may present a number of different forms. The crystals formed at the bottom may be different from those which are deposited on the side walls or at the surface.

If these concentration currents can completely change the habit of a crystal by producing etofigation in one direction, the nature of the faces is not modifled; an octahedral crystal always appears in octahedrons. But there are two other influetices which modify the faces. One of them is the rapidity of crystallization, the other the constant absorption of foreign matter by the crystal in process of growth. Still other factors may intervene, but they are only ndirectly concerned.

It has long been known that crystals formed rapidly possess simple faces, while those which have grown crystals rich in inclusions sometimes of large size, are poor in faces, while the small crystals of the same substance, but transparent, are generally limited by a large number of faces. These differences are due to the rate of crystallization, the influence of which has been known by the experiments of Frankenheim, Lecoq de Bosbaudran; O. Lehmann, and myself In rapid crystallization the crystals have faces with simple symbols; in slow crystallization these same simple faces persist, but the angles and edges have been truncated and beveled, giving rise to new facets, and I have shown that in certain cases these facets make their appearance always in the same order With vary. Ing rates of crystallization the dominant forms ob- ained in the case where the crystallization was rapid persist with more or less extensive development, but it may be otherwise in the case where the faces ar modified by the regular absorption by the crystal durng the growth of foreign matter added to the mother liquor. This fact is easily made evident, as I have demonstrated, by adding a little coloring matter.

Rome de l'Isle and Berniard have observed that the crystals of sodium chloride formed in urine are reguar octahedrons instead of cubes, such as crystallize rom a pure mother liquor. Vanquelin and Fourcroy showed later that this curious modification is due to the urea present. Boydant also established a few phenomena of the same class, and tried without success to ascertain why the mere presence of a foreign substance can be thus effective.

P. Curie developed a remarkable and attractive theory, which apparently furnished the key to this curious modification. He claims that the capillary action existing between the liquid and the crystal in tervenes, an effect varying with the nature of the laces belonging to the diverse forms and with the nature of the liquid. Basing his belief on Gauss's theory of capillarity, he concludes that such faces develop or require the minimum expenditure of caplllary energy. The dominant forms must consequently be conditioned by those faces the constant capillarity of which is the least. The addition of a foreign substance altering the different capillary constants may onsequently induce modiflcations of form.

It appears, indeed, that the capillary forces must act, but up to this time there is no fact known which proves that they intervene sufficiently to modify the orms, in spite of the experiments of M. Berent caried out in the laboratory of Sohncke; moreover, I shall describe later an observation showing that they are without influence.

The crystals of one substance rarely form synchron ously with those of another dissolved in the same mother liquid, and it is on this property that chemists base their action when they attempt to purify bodies by repeated crystallizations; but there are exceptions, as in the well-known coloration of hydrated nitrate of strontium by extract of logwood, which was accomplished by Senarmont. Since then M. Lehmann and $I$ have proved a few other cases of coloration of crystals by artiflcial organic dyes.

By making use of the artiflcial coloration of crystals so as to indicate the presence of foreign matter which has crystallized with the colorless substance have been enabled to show that the absorption caused modiflcation in form.

The absorption of foreign matter by crystals in process of formation is accomplished in two different ways: First, the coloring matter enters into the composition of the crystal, whatever may be its degree of dilution, and is shared betwein the crystal and the liquid; second, the coloring matter is taken up by the crystal only when the liquid becomes saturated.

The two procesess may go on simultaneously. The study of certain properties of colored crystals, particularly polychroism, and the law of division, shows that the coloring substance in the first case is found in the crystal in the same state as in the liquid; in the second, on the contrary, the coloring matter is in the crystalline state, and we have to do then with a regular grouping of the crystalline particles of the colorless substance with those of the coloring material added to the mother liquor.

Lead nitrate is colored by methylene blue in the econd manner; it appears in cubic crystals with the triglyphic striæ of pyrite instead of in octahedrons. The modiflcations in the crystals of this salt pro duced in a mother liquor which holds methylene blue n solution, show that capillarity does not interven to produdce them. Indeed, in a solution depositing lead nitrate and saturated with methylene blue, without, however, giving crystals of this latter substance, the crystals of the nitrate are not at all modifled. They are in octahedrons and colorless, but as soon as the coloring matter begins to crystallize synchronously with them the cube faces appear and finally are formed to the exclusion of all others. Nevertheless, the surface tension can not have been changed since the quantity of methylene blue has remained the same in solution. An interesting fact is the inequality of absorption of the foreign matter dissolved in the mother liquid.by the different faces of the crystal. Thus, on the octahedral faces of the lead nitrate the methylene blue is not at all deposited, but only on the faces of the cube and the pentagonal dodecahedron. Similar examples can be cited which explain the ap 
pearance, frequent in minerals, known as hour-glass structure. In the case of cubic crystals, of all the possible faces it is only those which absorb the foreign matter which will develop.

The idea which first comes to mind is that the molecular structure of the crystal plays an important part in this synchronous crystallization. It is not so at all; different foreign substances may be absorbed by different faces, and in such cases the habit of the crystal is dependent on these diverse substances. If one causes the colorless substance to crystallize in a solution containing two colors, each one giving characteristic forms, the crystallizations thus obtained will be the two combined forms, so that one and the same crystal is composed of pyramids or of prisms of different colors. Thus the crystals of urea nitrate colored by methylene blue and picric acid show, if the crystallization has been carefully conducted, yellow triangular prisms corresponding to the faces $g^{\prime}$ and $h^{\prime}$, and blue triangular prisms corresponding to the prismatic faces $m$ of the monoclinic system.

Not only may foreign crystalline matter be absorbed, but also the liquid matter added to the mother liquid, and even the molecules of the latter may also pass regularly into the crystal to modify its form. I have been able to show this fact by crystallizing phthalic acid in a solution containing ethyl alcohol." This explains why a crystal obtained from different solvents may show different faces.

Consequently a crystal, very pure in appearance, transparent, and without inclusions, may contain for

${ }^{1}$ To show this, it is enough to take a colored liquid, but with the exception of bromide there is no
proper color at the ordinary temperature. eign matter, and in the case where it is the mother liquid which is absorbed its purf

When the crystals of a determined substance obtained from two different solutions do not present the same forms, it is incontrovertible that in one of the cases, perhaps in both cases, since we do not always know the form of the pure crystal, there has been absorption of the molecules of the mother liquor. Some times it is the water which is absorbed, and this water has been regarded as water of crystallization or as water of constitution, according to the temperature at which it is driven off.

When purification is attempted by recrystallization, if the foreign substance which passes into the crysta is present in small quantities in the mother liquid, the first or the last crystals formed, according to the mode of synchronous crystallization, will be the purest. In case there is a diviston of the foreign matter between the crystal and the liquid, if the coefficient of its solubility in the crystal and the liquid are known, the number of crystallizations demanded for the purification of the crystals may be calculated under prope conditions.

The natural crystals appear in such varied habits that before Rome de l'Isle no one could see the constancy of forms, and the genius of Hauy was necessary to establish their derivation. It is known that ordinarily the crystals of the same deposit and the same generation are identical, and that those of successive deposits or generations may have different dominant forms. All these differences may be ex- plained by the rapidity of crystallization, but especially by the constant presence of foreign substances. Unfortunately it is difficult to determine the nature of the latter, since the results of analyses made up to the present time have little value in solving this problem. Indeed, a very small quantity of matter is rea forms of a crystal; sometime amount even less than one

In every case, whether we have to do with natural or artificial crystals, we need to determine their form in the pure state, a form which is constant and which I have called fundamental. It may be distinct from the primitive form chosen by crystallographers.

In closing, I shall observe that the substances pre pared in laboratories seem rarely to show the numerous modifications of form, so frequent in the natural crystals. This is due to the fact that the artificial crystals are prepared almost always in the same manner, with the same reagents and consequently with the same foreign substances in the mother liquor. In nature, on the contrary, as the analyses of mineral waters show, the composition of the solutions which deposit the crystals of a given substance varies from one region to another as much in the quality as in the quantity of the different elements dissolved. But all crystals do not lend themselves with the same facility to these modifications of the faces; and just as there exist in nature bodies like calcite which possess the most varied habits, there exist also artificial compounds, the crystals of which may appear in a great number of forms, depending on the condition of crystallization, as, for example, phthalic acid, meconic acid, nitrate and oxalate of urea.

\section{Lord Morley on Science and Literature}

\section{Association with Science as a Literary Asset-The Literary Shortcomings of Scientific Men}

An eloquent address on language and literature was delivered on January 27th by Lord Morley of Blackburn, as president of the English Association. Parts of the address dealt with the relation between science and letters, with particular reference to the use of scientific knowledge in poetry, and the antithesis between documentary fact and artistic style. Science aims at concise and truthful expression; and while Lord Morley testified to the value of its influence upon literature, he doubted whether scientific ideas had inspired even Tennyson to the best verse, whether the desire for fact scientifically recorded is not a misfortune in the treatment of modern history, and whether concect upon emotional conceptions and pleasures.

In commenting on the address, Nature remarks: "Lord Morley's tribute to some scientific master of clear and simple exposition resigns us to his subsequent conclusions. Keats could not forgive optics for robbing the rainbow of its wonder and mystery, and Lord Morley seems to suggest that the literary art which deals with scientific studies and results is not of the highest. But poetry is imagery, and new im ages of Nature are made possible by every discovery of the attributes and meaning of the things around us The poetry which neglects advances of natural knowl edge becomes conventional in form and substance, concerning itself only with the wonders of childhood because it does not understand the higher and grander mysteries which science has failed to penetrate. History is concerned with the accumulation and consideration of facts with the view of arriving at cor rect conclusions from them; and in this respect must be studied by the methods of science, though the human factor makes the problems more difficult than when material things only are involved. There is however, no intrinsic reason why Gibbon's majesty of historic conceptions and the symmetric grandeur of his design should not be combined with such great learning as was displayed by Lord Acton. Accurate knowledge must surely not be considered as antithetic to perfection of style.

"The instance of Darwin's loss of interest in poetry and music proves little. A wide search through th biographies of distinguished men of science will onl reveal two or three cases in which devotion to studie of Nature has resulted in the atrophy of æsthetic faculties. Close concentration upon any particular subject often leads to indifference to the aims and work of others; but this is as true of art, or poetry, or music, as it is of science. There is less reason for be lieving that the man of science has usually no tast for literature, music, or other forms of refined and imaginative expression, than there is for concludin that artists, musicians, and poets have no interest in the attentive study of natural objects and phenomena. If science and documentary evidence are responsible for an age of prose, it is because the poets have bee spinning cobwebs from their brains when they ought to have boen learning something of the splrit and achievements of science. These are they who, having never entered upon scientific pursuits, are, to use Herbert Spencer's words, 'blind to most of the poetry by which they are surrounded.'

Subjoined are some extracts:

Let me offer a few words on the effects of the relations of letters and science. We may obviously dat a new time from 1859 v'hen Darwin's "Origin of Species" appeared, and along with two or three othe imposing works of that date launched into commo currency a new vocabulary. We now apply in ever sphere, high and low, trivial or momentous, talk about evolution, natural selection, environment, he redity, survival of the fittest, and all the rest. The most resolute and trenchant of Darwinians has warned us that new truths begin as rank heresies and end as superstitions; and if he were alive to see to day all the effects of his victory on daily speech, pe haps he would not withdraw his words. That great controversy has died down, or at least takes new shape, leaving, after all is said, one of the master contributions to knowledge of nature and its laws and to man's view of life and the working of his destinies.

Scientific interest has now shifted into new areas of discovery, invention, and speculation. Still the spirit of the time remains the spirit of science, and fact and ordered knowledge. What has been the effect of knowledge upon form, or language, on literary art? It adds boundless gifts to human conveniences. Does it. make an inspiring public for the master of either prose or verse? Darwin himself made no pretensions in authorship. He once said to Sir Charles Lyell that a naturalist's life would be a happy one if he had only to observe and never to write. Yet he is a writer patient accumulation of persuasive arguments a noble and transparent candor in stating what makes against him, which, if not what is called style, is better for the reader than the finest style can be. One eminent literary critic of my acquaintance finds his little volume on earthworms a most fascinating book even as literature. Then, although the controversial exigencies of his day affected him with a relish for laying too lustily about him with his powerful flail, I know no more lucid, effective, and manful English than you will find in Huxley. What more delightful book of travel than the "Himalaya Journals" of the great naturalist Hooker, who carried on his botanical explorations some sixty years ago, and happily is still among us?

Buffon, as man of science, is now, I assume, little more than a shadow of a name, and probably even the most highly educated of us know little more about him than his famous pregnant saying that the style is the man-a saying, by the way, which really meant no more than that, while nature gave the material for narrative, it is man who gives the style. Yet the French to this day count him among the greatest of their writers for order, unity, precision, method, clearness in sclentific exposition of animated nature, along with majeetic gifts of natural eloquence. Then comes the greatest of all. Whatever the decision may as to the value of Goethe's scientiflc contribution, this, at least, is certain, that his is the most wondrous, the unique case of a man who united high original scientific power of mind with transcendent gifts in filght, force, and beauty of poetic imagination.

As for science and the poets, only the other day an attractive little book published by Sir Norman Lockyer shows how Tennyson, the composer of verse unsurpassed for exquisite music in our English tongue, yet followed with unflagging interest the problems of evolution and all that hangs upon them. Whether astronomy or geology-terrible muses, as he well might call them-inspired the better elements of his beautiful work, we may doubt. An English critic has had the courage to say that there is an insoluble element of prose in Dante, and Tennyson has hardly shown that the scientific ideas of an age are soluble n musical words. Browning, his companion poet. nearly universal in his range, was too essentially dramatic, too independent of the scientific influences of his day, too careless of expression, to be a case much in point. Tennyson said of him, he had power of intellect enough for all of them "but he has not the glory of words." Whether he had or not, science was not responsible.

I should like to name in passing the English poet who, in Lowell's words, has written less and pleased more than any other. Gray was an incessant and a serious student in learned tongues; and his annotations on the "System of Nature," by Linnæus, his contemporary, bear witness to his industry and minute observation as naturalist.

In prose fiction was one writer of commanding mind, saturated with the spirit of science. Who does not feel how George Eliot's creative and literary art was impaired, and at last worse than impaired, by her daily associations with science? Or would it be truer to say-I often thought it would-that the decline was due to her own ever-deepening sense of the pain of the world and the tragedy of sentient being? Let us look at the invasion of another province by the spirit of the time. The eager curiosity of all these and their laws has inevitably quickened the spread both of the same curiosity and the same respect quickened by German example, for ascertained facts into the province of history. Is the pure scientific impulse-to tell the exact truth with all the necessary
reservations-easy to combine with regard for artistic pleasure?

The English writer of our own immediate time, with the fullest knowledge and deepest understanding of the fact and spirit of history, would, I think, be pronounced by most critics with a right to judge, to be the late Lord Acton. Acton's was a leading case form. His page is overloaded, he is often over-subtle he has the fault-or shall I call it the literary crime? $\rightarrow$ of allusiveness and indirect reference-he is apt to put to his reader a riddle or a poser, and then to leave 